EESTI NSV TEADUSTE AKADEEMIA TOIMETISED, 32. KOIDE

FOOSIKA * MATEMAATIKA, 1983, NR. 3

ИЗВЕСТИЯ АКАДЕМИИ НАУК ЭСТОНСКОН ССР, ТОМ 32 ФИЗНКА * МАТЕМАТИКА. 1983, № 3

\title{
ПРИБЛИЖЕННО МИНИМАКСНАЯ ОЦЕНКА СРЕДНЕГО ОДНОМЕРНОГО НОРМАЛЬНОГО РАСПРЕДЕЛЕНИЯ
}

\author{
(Представил Н. Алумяэ)
}

\section{Введение}

Пусть $X$ - одномерная случайная величина, распределенная по нормальному закону $N\left(\mu, \sigma^{2}\right)$ с дисперсией $\sigma^{2}=1$ и средним $\mu$, пе превышающим по модулю известной положительной константы $a$, т. е. $\mu \in[-a, a]$. Рассмотрим проблему построения оценки среднего $\mu$, минимизирующей максимум риска при квадратичной потере. Известно, что минимаксная оценка является байесовской оценкой относительно апрнорного распределения на интервале $[-a, a]$, при котором байесовский риск максимален. Тем самым построение минимаксной оценки сводится к нахождению априорного распределения, максимизирующего байесовский риск.

В [ ${ }^{1}$ ] показано, что априорное распределение с максимальным байесовским риском, являющееся симметричным и единственным, при $a \leqslant 1,05$ сосредоточено в граничных точках интервала $[-a, a]$, а при $a \leqslant 2-$ в этих же точках и в нуле. Вероятности точек сосредоточения при каждом $a=1,1(0,1) 2$ найдены численным путем. В $\left[{ }^{2}\right]$ построены оценки среднего $\mu$, являющиеся при $a \rightarrow \infty$ асимптотически минимаксными второго порядка на интервале $[-a, a]$. Такие же оценки рассмотрены в $\left[{ }^{3}\right]$.

В настоящей статье приводятся построенные автором численными методами априорные распределения на интервале - $[-a, a]$ при $a=1,1(0,1) 4(0,2) 6(0,4) 8(1) 10$, обладающие почти максимальным байесовским риском. Максимальный риск соответствующих байесозских оценок с точностью проведенных вычислений совпадает с байесозским риском. Поскольку максимальный риск не может быть меньше байесовского риска, то эти байесовские оценки являются приближенно минимаксными, и с точки зрения применений проблема построения минимаксных оценок решена для вышеуказанных значений $a$. Приводимые результаты до $a=2$ совпадают с результатами $\left[{ }^{1}\right]$, за исключением случая, когда $a=1,5$. Есть основания надеяться, что построенные априорные распределения близки к наименее благоприятным не только в смысле байесовского риска, но и в смысле точек сосредоточения распределения и вероятностей этих точек.

\section{Постановка задачи и известные предложения}

Пусть $X \sim N(\mu, 1)$, где $\mu$ - число из заданного интервала $[-a, a]$. Для оценки $d(X)$ среднего $\mu$ определим функцию риска в виде

$$
R(d, \mu)=(2 \pi)^{-1 / 2} \int_{-\infty}^{+\infty}[d(x)-\mu]^{2} \mathrm{e}^{-(x-\mu)^{2} / 2} \mathrm{~d} x .
$$


Задача заключается в построении минимаксной оценки $d_{a}^{*}(X)$, минимизирующей функционал

$$
q_{a}(d)=\sup _{\mu \in[-a, a]} R(d, \mu) .
$$

Пусть $\Xi_{a}-$ класс априорных вероятностных мер $\xi$, определенных на б-алгебре борелевских подмножеств интервала $[-a, a], r_{\xi}-$ байесовский риск, соответствующий априорному распределению $\xi$, т. е.

$$
r_{\xi}=\inf _{d-a} \int_{-a}^{a} R(d, \mu) \mathrm{d} \xi(\mu)
$$

a $d_{\xi}(X)$ - байесовская оценка относительно априорного распределения $\xi$, минимизирующая функционал $\int_{-a}^{a} R(d, \mu) \mathrm{d} \xi(\mu)$. Пусть $\xi^{*}{ }_{a}-$ наименее благоприятное распределение в классе $\Xi_{a}$, т. е. такое, что

$$
r_{\xi_{a}}=\sup _{\xi \in \Sigma_{a}} r_{\xi} .
$$

Приведем здесь некоторые известные предложения, имеющие отношение к построению минимаксной оценки.

Предложение 1. Существует наименее благоприятное распределенuе в классе $\Xi_{a}$.

Предложение 2. Наименее благоприятное распределение $\xi_{a}^{*}$ в классе $\Xi_{a}$ сосредоточено на мнонестве точек интервала [-a; $]$, где байесовская функция риска $R\left(d_{\text {s: }^{*}}, \cdot\right)$ достигает максимального значенuя.

Предложение 3. Наименее благоприятное распределение в классе $\Xi_{a}$ сосредоточено на конечном множестве точек.

Предложени е 4. Наименее благоприятное распределение в классе $\Xi_{a}$ присваивает равные вероятности симметричным относительно нуля точкам.

Предложение 5. Наименее благоприятное распределение в классе $\Xi_{a}$ единственно.

Предложение 6. Существует минимаксная оценка $d_{a}^{*}(X), u$ она является байесовской оценкой относительно наименее благоприятного распределения в классе $\Xi_{a}$.

Пр едложен и е 7. Байесовская оценка относительно любого априорного распределения छे из класса $\Xi_{a}$ задается на всей прямой (исключая, быть может, множество лебеговской меры нуль) следующзей формулой:

$$
d_{\xi}(x)=\frac{\int^{a} \mu \mathrm{e}^{-(x-\mu)^{2} / 2} \mathrm{~d} \xi(\mu)}{\int^{a} \mathrm{e}^{-(x-\mu)^{2 / 2}} \mathrm{~d} \xi(\mu)}
$$

Предложение $8\left(\left[{ }^{4}\right]\right)$. Если $\xi^{1}, \xi^{2}, \ldots$ - такая последовательность априорных распределений в классе $\Xi_{a}$, что соответствующая последовательность байесовских рисков $r_{\xi^{1}}, r_{\xi^{2}}, \ldots$ сходится $\kappa$ максимальному байесовскому риску $r_{\xi_{a}^{*}}$, то последовательность байесовских функций риска $R\left(d_{\xi^{\prime}}, \cdot\right), R\left(d_{\xi^{2}}, \cdot\right), \ldots$ сходится равномерно на интервале $[-a, a]$ $\kappa$ единственной минимаксной функции риска $R\left(d_{\alpha}{ }^{*}, \cdot\right)$. 


\section{О процедуре вычислений}

На выбор процедуры вычислений в настоящей работе значительно повлияли идеи работы $\left[{ }^{4}\right]$, где для построения минимаксной оценки предложен итерационный метод. Однако стремление обойтись малым количеством вычислительных операций, а также условия работы с ЭВМ «Минск-32», имеющей небольшой объем оперативной памяти, привели к построению нового алгоритма для решения конкретной задачи. Итерационным методом ищется априорное распределение с «большим» байесовским риском, что с учетом предложения 8 дает надежду на «малость» максимума соответствующей байесовской функции риска $R\left(d_{\xi}, \cdot\right)$ на интервале $[-a, a]$. В силу предложений 3 и 4 было разумно построить процедуру поиска на симметричных распределениях, сосредоточенных на конечном множестве точек. Предложения 2 и 8 указывают на то, что при выполнении итераций следует точки сосредоточения распределения сдвигать в сторону возрастания байесовской функции риска, а вероятности этих точек выбирать так, чтобы байесовская функция риска принимала в них одно и то же значение. Последнего естественно добиваться увеличением априорных вероятностей в точках, где байесовская функция риска имеет большие значения.

При разработке алгоритма было сделано предположение, что если для некоторого $а$ наименее благоприятное распределение $\xi_{a}{ }^{*}$ сосредоточено в точках $\pm c_{a 1}^{*}, \ldots, \pm c_{a m_{a}-1}^{*}, \pm a$, то существуют неотрицательные числа $b_{1}$ и $b_{2}$ такие, что при $0 \leqslant \Delta a \leqslant b_{1}$ распределение $\xi_{a+\Delta a}^{*}$ сосредоточено в точках

$$
\pm\left[\left|c_{a 1}^{*}\right|+\delta_{a 1}(\Delta a)\right], \ldots, \pm\left[\left|c_{a m_{a}-1}^{*}\right|+\delta_{a m_{a}-1}(\Delta a)\right], \pm[a+\Delta a]
$$

а при $b_{1}<\Delta a \leqslant b_{2}-$ в точках

$$
0, \pm\left[\left|c_{a 1}^{*}\right|+\delta_{a 1}(\Delta a)\right], \ldots, \pm\left[\left|c_{a m_{a}-1}^{*}\right|+\delta_{a m_{a}-1}(\Delta a)\right], \pm[a+\Delta a],
$$

где $\delta_{a 1}(\Delta a), \ldots, \delta_{a m_{a}-1}(\Delta a)$ - непрерывные монотонно неубывающие функции. Другими словами, при расширении интервала $[-a, a]$ точки сосредоточения наименее благоприятного распределения непрерывно удаляются от нуля, а в нуле появляется новая точка сосредоточения распределения, которая затем раздваивается.и т. д. Результаты вычислений подтвердили справедливость такого предположения. Таким образом, если при некотором $a=a_{0}$ находилось распределение $\xi_{a_{\circ}}$ с байесовским риском, близким к максимальному, то при $a=a_{0}+\Delta a$ в качестве начального приближения выбиралось распределение, сосредоточенное в точках, близких к тем, в которых сосредоточено распределение $\xi_{a_{0}}$. Аналогично выбирались и вероятности этих точек. Вычисления начинались с $a=1$, когда наименее благоприятное распределение сосредоточено в точках -1 и +1 , и продолжались до $a=10$.

\section{Результаты вычислений}

Для каждого промежутка $[-a, a]$ при $a=1,1(0,1) 4(0,2) 6(0,4) 8(1) 10$ определено распределение $\xi_{a}$ с почти максимальным байесовским риском $r_{\xi_{a}} \approx r_{\xi_{a}^{*}}, \quad$ присваивающее точкам $\pm c_{a 1}, \ldots, \pm c_{a m_{a}}$ соответственно вероятности $\xi_{a 1} / 2, \ldots, \xi_{a m_{a}} / 2$, где $\sum_{j=1}^{m_{a}} \xi_{a j}=1$. В таблице приведены для каждого $a$ модули $+c_{a 1}, \ldots,+c_{a m_{a}}$ точек сосредоточения распределения $\xi_{a}$, двукратные вероятности этих точек $\xi_{a 1}, \ldots, \xi_{a m_{a}}$ и 
Априорные распределения $\xi_{a}$ с байесовским риском, близким к максимальному

\begin{tabular}{l|l|l}
\hline$a$ & $q_{a}\left(d_{\xi_{a}}\right) \mid+c_{a j}$ & $\xi_{a j}$ \\
\hline
\end{tabular}

\begin{tabular}{l|l|l}
\hline$a\left|q_{a}\left(d_{\xi_{a}}\right)\right|+c_{a j}$ & $\xi_{a j}$ \\
\hline
\end{tabular}

\begin{tabular}{|c|c|c|c|}
\hline 1,1 & 0,471 & $\begin{array}{l}0 \\
1,1\end{array}$ & $\begin{array}{l}0,0499 \\
0,9501\end{array}$ \\
\hline 1,2 & 0,492 & $\begin{array}{l}0 \\
1,2\end{array}$ & $\begin{array}{l}0,1435 \\
0,8565\end{array}$ \\
\hline 1,3 & 0,513 & $\begin{array}{l}0 \\
1,3\end{array}$ & $\begin{array}{l}0,2145 \\
0,7855\end{array}$ \\
\hline 1,4 & 0,535 & $\begin{array}{l}0 \\
1,4\end{array}$ & $\begin{array}{l}0,2692 \\
0,7308\end{array}$ \\
\hline 1,5 & 0,556 & $\begin{array}{l}0 \\
1,5\end{array}$ & $\begin{array}{l}0,3117 \\
0,6883\end{array}$ \\
\hline 1,6 & 0,577 & $\begin{array}{l}0 \\
1,6\end{array}$ & $\begin{array}{l}0,3450 \\
0,6550\end{array}$ \\
\hline 1,7 & 0,596 & $\begin{array}{l}0 \\
1,7\end{array}$ & $\begin{array}{l}0,3711 \\
0,6289\end{array}$ \\
\hline 1,8 & 0,615 & $\begin{array}{l}0 \\
1,8\end{array}$ & $\begin{array}{l}0,3916 \\
0,6084\end{array}$ \\
\hline 1,9 & 0,631 & $\begin{array}{l}0 \\
1,9\end{array}$ & $\begin{array}{l}0,4077 \\
0,5923\end{array}$ \\
\hline 2,0 & 0,645 & $\begin{array}{l}0 \\
2,0\end{array}$ & $\begin{array}{l}0,4204 \\
0,5796\end{array}$ \\
\hline 2,1 & 0,657 & $\begin{array}{l}0,361 \\
2,1\end{array}$ & $\begin{array}{l}0,4585 \\
0,5415\end{array}$ \\
\hline 2,2 & 0,670 & $\begin{array}{l}0,502 \\
2,2\end{array}$ & $\begin{array}{l}0,4926 \\
0,5074\end{array}$ \\
\hline 2,3 & 0,681 & $\begin{array}{l}0,602 \\
2,3\end{array}$ & $\begin{array}{r}0,5221 \\
-0,4779\end{array}$ \\
\hline 2,4 & 0,693 & $\begin{array}{l}0,681 \\
2,4\end{array}$ & $\begin{array}{l}0,5473 \\
0,4527\end{array}$ \\
\hline 2,5 & 0,704 & $\begin{array}{l}0,747 \\
2,5\end{array}$ & $\begin{array}{l}0,5687 \\
0,4313\end{array}$ \\
\hline 2,6 & 0,715 & $\begin{array}{l}0,804 \\
2,6\end{array}$ & $\begin{array}{l}0,5868 \\
0,4132\end{array}$ \\
\hline
\end{tabular}

$\begin{array}{llll}3,5 & 0,787 & 0 & 0,2523 \\ & & 1,645 & 0,4649 \\ & & 3,5 & 0,2828 \\ 3,6 & 0,793 & 0 & 0,2615 \\ & & 1,718 & 0,4656 \\ & & 3,6 & 0,2729\end{array}$

$\begin{array}{llll}3,7 & 0,799 & 0 & 0,2687 \\ & & 1,786 & 0,4672 \\ & 3,7 & 0,2641\end{array}$

$3,8 \quad 0,805 \quad 0,313 \quad 0,2974$

$\begin{array}{lll}1,887 & 0,4483 \\ & 3,8 & 0,2543\end{array}$

$\begin{array}{llll}3,9 & 0,810 & 0,487 & 0,3350 \\ & 2,008 & 0,4212 \\ & 3,9 & 0,2438\end{array}$

$\begin{array}{llll}4,0 & 0,815 & 0,598 & 0,3668\end{array}$

\begin{tabular}{llll} 
& & 2,126 & 0,3996 \\
4,2 & \multirow{4}{*}{0,825} & 0,730 & 0,4094 \\
& & 2,334 & 0,3749 \\
& & 4,2 & 0,2157 \\
4,4 & 0,834 & 0,815 & 0,4358 \\
& & 2,514 & 0,3634 \\
& & 4,4 & 0,2008
\end{tabular}

$\begin{array}{llll}4,6 & 0,842 & 0 & 0,0372 \\ & 0,937 & 0,4210 \\ & 2,684 & 0,3536 \\ & 4,6 & 0,1882\end{array}$

$\begin{array}{llll}4,8 & 0,850 & 0 & 0,1455 \\ & 1,268 & 0,3581 \\ & 2,909 & 0,3220 \\ & 4,8 & 0,1744\end{array}$

$\begin{array}{llll}5,0 & 0,857 & 0 & 0,1779 \\ & 1,463 & 0,3584 \\ & 3,116 & 0,3013 \\ & 5,0 & 0,1624\end{array}$

$\begin{array}{llll}2,8 & 0,734 & 0,901 & 0,6151 \\ & & 2,8 & 0,3849 \\ 2,9 & 0,743 & 0 & 0,0040 \\ & & 0,947 & 0,6222 \\ & & 2,9 & 0,3738\end{array}$

$\begin{array}{llll}3,0 & 0,751 & 0 & 0,1122 \\ & & 1,121 & 0,5333 \\ & 3,0 & 0,3545\end{array}$

$\begin{array}{llll}5,2 & 0,864 & 0 & 0,1937 \\ & & 1,602 & 0,3660 \\ & & 3,307 & 0,2883 \\ & & 5,2 & 0,1520 \\ 5,4 & 0,870 & 0,278 & 0,2196 \\ & & 1,749 & 0,3600 \\ & & 3,490 & 0,2776 \\ 5,6 & 0,876 & 5,4 & 0,1428 \\ & & 0,585 & 0,2849 \\ & & 3,010 & 0,3234 \\ 5,8 & 0,881 & 5,6 & 0,2582 \\ & & 0,712 & 0,1335 \\ & & 2,224 & 0,3225 \\ & & 3,908 & 0,2423 \\ 6,0 & 0,886 & 0,782 & 0,1251 \\ & & 2,387 & 0,3426 \\ & & 4,101 & 0,3083 \\ & & 6,0 & 0,1177 \\ & & & \end{array}$




\begin{tabular}{|c|c|c|c|c|c|c|c|}
\hline$a$ & $q_{a}\left(d_{\xi_{a}}\right)$ & $+c_{a j}$ & $\xi_{a j}$ & $a$ & $q_{a}\left(d_{\xi_{a}}\right)$ & $+c_{a j}$ & $\xi_{a j}$ \\
\hline 6,4 & 0,896 & $\begin{array}{l}0 \\
1,275 \\
2,795 \\
4,500 \\
6,4\end{array}$ & $\begin{array}{l}0,1270 \\
0,2842 \\
0,2768 \\
0,2077 \\
0,1043\end{array}$ & 8,0 & 0,923 & $\begin{array}{l}0 \\
1,336 \\
2,793 \\
4,379 \\
6,097 \\
8,0\end{array}$ & $\begin{array}{l}0,1179 \\
0,2419 \\
0,2351 \\
0,1988 \\
0,1392 \\
0,0671\end{array}$ \\
\hline 6,8 & 0,904 & $\begin{array}{l}0 \\
1,559 \\
3,174 \\
4,897 \\
6,8\end{array}$ & $\begin{array}{l}0,1574 \\
0,3018 \\
0,2601 \\
0,1878 \\
0,0929\end{array}$ & \multirow[t]{2}{*}{9,0} & \multirow[t]{2}{*}{0,935} & \multirow{2}{*}{$\begin{array}{l}0,738 \\
2,228 \\
3,764 \\
5,373 \\
7,096 \\
9,0\end{array}$} & \multirow{2}{*}{$\begin{array}{l}0,2420 \\
0,2293 \\
0,2030 \\
0,1626 \\
0,1107 \\
0,0524\end{array}$} \\
\hline 7,2 & 0,911 & $\begin{array}{l}0,634 \\
2,021\end{array}$ & $\begin{array}{l}0,2501 \\
0,2631\end{array}$ & & & & \\
\hline & & $\begin{array}{l}3,585 \\
5,298 \\
7,2\end{array}$ & $\begin{array}{l}0,2347 \\
0,1691 \\
0,0830\end{array}$ & \multirow[t]{2}{*}{10,0} & \multirow[t]{2}{*}{0,945} & \multirow{2}{*}{$\begin{array}{l}0 \\
1,551 \\
3,113 \\
4,705 \\
6,349 \\
8,089 \\
10,0\end{array}$} & \multirow{2}{*}{$\begin{array}{c}0,1181 \\
0,2288 \\
0,2085 \\
0,1767 \\
0,1362 \\
0,0899 \\
0,0418\end{array}$} \\
\hline 7,6 & 0,917 & $\begin{array}{l}0,774 \\
2,341 \\
3,966 \\
5,695 \\
7,6\end{array}$ & $\begin{array}{l}0,2871 \\
0,2648 \\
0,2198 \\
0,1538 \\
0,0745\end{array}$ & & & & \\
\hline
\end{tabular}

максимум функции риска при байесовской оценке $q_{a}\left(d_{\xi_{a}}\right)=\sup _{\mu \in[-a, a]}$ $R\left(d_{\xi_{a}}, \mu\right)$. В таблице значения функционала $q_{a}\left(d_{\xi_{a}}\right)$ приведены с точностью до трех знаков после запятой, а вычисления проводились с точностью до четырех знаков без учета погрешностей вычисления интеграла при определении значений функции риска $R\left(d_{\xi a}, \cdot\right)$.

При вычислении значений функции $R\left(d_{\text {sa }}, \cdot\right)$ использовалась квадратурная формула наивысшей алгебраической степени точности для интегрирования по оси $-\infty<x<+\infty$ с весом exp $\left(-x^{2}\right)$. Вычисления проводились по формуле, точной для многочленов порядка 35 , и проверялись по формуле, точной для многочленов порядка $33\left[{ }^{5}\right]$. Результаты вычислений по обеим формулам совпали с точностью до четвертого знака после запятой.

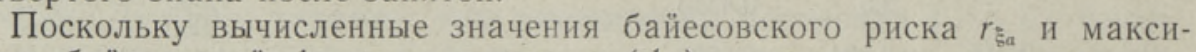
мума байесовской функции риска $q_{a}\left(d_{\xi_{a}}\right)$ совпали с точностью до четвертого знака после запятой, а для минимаксной оценки $d_{a}{ }^{*}(X)$ имеют место неравенства

$$
r_{\xi_{a}} \leqslant q_{a}\left(d_{a}^{*}\right) \leqslant q_{a}\left(d_{\xi_{a}}\right),
$$

то в пределах точности проведенных вычислений решением поставленной задачи, т. е. минимаксной оценкой, можно считать байесовскую оценку $d_{\xi a}(X)$. По предложению 7 эта оценка представима в виде

$$
d_{\xi_{a}}(X)=\frac{\sum_{j=1}^{m_{a}}+c_{a j} \xi_{a j} \mathrm{e}^{-c_{a j}^{2} / 2}\left(\mathrm{e}^{+c_{a j} X}-\mathrm{e}^{-c_{a j} X}\right)}{\sum_{j=1}^{m_{a}} \xi_{a j} \mathrm{e}^{-c_{a j}^{2} / 2}\left(\mathrm{e}^{+c_{a j} X}-\mathrm{e}^{-c_{a j} X}\right)}
$$

где для фиксированного значения a соответствующие числа $+c_{a j}$ и и $\xi_{a j}\left(j=1, \ldots, m_{a}\right)$ выбираются из таблицы.

\section{Заключение}

Если задача состоит в построении минимаксной оценки среднего $\mu$ по выборке $X_{1}, \ldots, X_{n}$ из нормального распределения $N\left(\mu, \sigma^{2}\right)$ 
с известной дисперсией $\sigma^{2}$ при условии, что $\mu$ принадлежит заданному интервалу $\left[a_{1}, a_{2}\right]$, то эта задача сводится к вышеизложенной в силу того, что $\bar{X}=\sum_{i=1}^{n} X_{i} / n$ является достаточной статистикой. Принимая $a=\left(a_{2}-a_{1}\right) n^{1 / 2} /(2 \sigma)$ и $\mu_{0}=\left(a_{1}+a_{2}\right) / 2$, можно записать оценку, прибллженно минимизирующую максимум риска при квадратичной потере, в следующем виде:

$$
\text { - } d_{a_{1}, a_{2}, \sigma}\left(X_{1}, \ldots, X_{n}\right)=\mu_{0}+\left(\sigma / n^{1 / 2}\right) d_{\xi_{a}}\left(\left(\bar{X}-\mu_{0}\right) n^{1 / 2} / \sigma\right) \text {. }
$$

В настоящей работе построены минимаксные оценки при $a \leqslant 10$ с достаточной для приложений точностью. Ранее такие оценки при $a \leqslant 2$ были получены в $\left.{ }^{1}\right]$. При больших $a$ можно пользоваться асимптотически минимаксными оценками, предложенными в $\left[{ }^{2,3}\right]$. Представление о том, насколько минимаксная оценка лучше обычно используемой оценки $\bar{X}$ с постоянной функцией риска $R(\bar{X}, \cdot)=\sigma^{2} / n$, можно получить, используя для сравнения значения максимального риска из таблицы, умноженные на $\sigma^{2} / n$. В случае $a=1,1$ минимальный выигрыш от использования минимаксной оценки, измеряемый относительным уменьшением риска, составляет $53 \%$. С возрастанием $a$ он, естественно, монотонно уменьшается и при $a=10$ равняется $5,5 \%$.

\section{Л И ТЕРА Т У Р А}

1. Casella, G., Strawderman, W. Ann. Statist., 9, № 4, 870-878 (1981).

2. Левит Б. Я. Теория вероятностей и ее применения, 25, № 3, 561-576 (1980).

3. Bickel, P. J. Ann. Statist., 9, № 6, 1301-1309 (1981).

4. Nelson, W. Ann. Math. Statist., 37, № 6, 1643-1657 (1966).

5. Крылов В. И., Шульгина Л. Т. Справочная книга по численному интегрированию. М., «Наука», 1966.
Институт кибернетики
Академии наук Эстонской ССР
Поступила в редакцию
30 сентября 1982

\section{J. KUKS}

\section{OHEMÕ̃TMELISE NORMAALJAOTUSE KESKVÄÄRTUSE LIGIKAUDNE MINIMAKSHINNANG}

Olgu $X_{1}, \ldots, X_{n}$ valim ühemõõtmelisest normaaljaotusest $N\left(\mu, \sigma^{2}\right)$, mille dispersioon $\sigma^{2}$ on teada ja keskväärtus $\mu$ kuulub etteantud vahemikku $\left[a_{1}, a_{2}\right]$. Numbrilisel teel on leitud keskväärtuse $\mu$ ligikaudne minimakshinnang $d_{a_{1}, a_{2}, \sigma}\left(X_{1}, \ldots, X_{n}\right)$ neil juhtudel, kui $\left(a_{2}-a_{1}\right) n^{1 / 2} /(2 \sigma) \leqslant 10$.

\section{J. KUKS}

\section{AN APPROXIMATELY MINIMAX ESTIMATOR OF THE MEAN OF AN ONE-DIMENSIONAL NORMAL DISTRIBUTION}

Let $X_{1}, \ldots, X_{n}$ be a sample from one-dimensional normal distribution $N\left(\mu, \sigma^{2}\right)$ with known variance $\sigma^{2}$ and unknown mean $\mu$ that belongs to a given interval. The problem considered is to construct a minimax estimator of the parameter $\mu$ in the case of quadratic loss.

As $\vec{X}=\sum_{i=1}^{n} X_{i} / n$ is a sufficient statistic, so the problem reduces to a simpler form where $\sigma^{2}=1, n=1$, and $a_{1}=-a_{2}$. An approximately minimax solution has been numerically found by an iterative procedure in the cases when $\left(a_{2}-a_{1}\right) n^{1 / 2} /(2 \sigma)=$ $=1.1(0.1) 4(0.2) 6(0.4) 8(1) 10$. The maximum risk of constructed estimators differs from minimax risk no more than 0.01 per cent and it is lower than the risk of the usual estimator $\bar{X}$, for example about 53 per cent if $\left(a_{2}-a_{1}\right) n^{1 / 2} /(2 \sigma)=1.1$, and about 5.5 per cent if $\left(a_{2}-a_{1}\right) n^{1 / 2} /(2 \sigma)=10$. 